\title{
Determination of One Unknown Thermal Coefficient through a Mushy Zone Model with a Convective Overspecified Boundary Condition
}

\author{
Andrea N. Ceretani ${ }^{1,2}$ and Domingo A. Tarzia ${ }^{1}$ \\ ${ }^{1}$ CONICET, Departamento de Matemática, Facultad de Ciencias Empresariales, Universidad Austral, Paraguay 1950, \\ S2000FZF Rosario, Argentina \\ ${ }^{2}$ Departamento de Matemática, Facultad de Ciencias Exactas, Ingeniería y Agrimensura, Universidad Nacional de Rosario, \\ Pellegrini 250, S2000BTP Rosario, Argentina
}

Correspondence should be addressed to Andrea N. Ceretani; aceretani@austral.edu.ar

Received 8 May 2015; Accepted 9 June 2015

Academic Editor: Mohsen Torabi

Copyright (C) 2015 A. N. Ceretani and D. A. Tarzia. This is an open access article distributed under the Creative Commons Attribution License, which permits unrestricted use, distribution, and reproduction in any medium, provided the original work is properly cited.

\begin{abstract}
A semi-infinite material under a solidification process with the Solomon-Wilson-Alexiades mushy zone model with a heat flux condition at the fixed boundary is considered. The associated free boundary problem is overspecified through a convective boundary condition with the aim of the simultaneous determination of the temperature, the two free boundaries of the mushy zone and one thermal coefficient among the latent heat by unit mass, the thermal conductivity, the mass density, the specific heat, and the two coefficients that characterize the mushy zone, when the unknown thermal coefficient is supposed to be constant. Bulk temperature and coefficients which characterize the heat flux and the heat transfer at the boundary are assumed to be determined experimentally. Explicit formulae for the unknowns are given for the resulting six phase-change problems, besides necessary and sufficient conditions on data in order to obtain them. In addition, relationship between the phase-change process solved in this paper and an analogous process overspecified by a temperature boundary condition is presented, and this second problem is solved by considering a large heat transfer coefficient at the boundary in the problem with the convective boundary condition. Formulae for the unknown thermal coefficients corresponding to both problems are summarized in two tables.
\end{abstract}

\section{Introduction}

Heat transfer problems with a phase-change such as melting and freezing have been studied in the last century due to their wide scientific and technological applications. Some books in the subject are [1-9].

In this paper we consider a phase-change process for a semi-infinite material, which is characterized by $x>0$, that is initially assumed to be liquid at its melting temperature (which without loss of generality we assume to be equal to $0^{\circ} \mathrm{C}$ ). We consider this material under a solidification process with the presence of a zone where solid and liquid coexist, known as "mushy zone," with a heat flux boundary condition imposed at the fixed face $x=0$. We follow $[10,11]$ in considering three different regions in this type of solidification process:

(1) Liquid region at temperature $T(x, t)=0: D_{l}=$ $\left\{(x, t) \in \mathbb{R}^{2} / x>r(t), t>0\right\}$.

(2) Solid region at temperature $T(x, t)<0: D_{s}=\{(x, t) \in$ $\left.\mathbb{R}^{2} / 0<x<s(t), t>0\right\}$.

(3) Mushy region at temperature $T(x, t)=0: D_{p}=$ $\left\{(x, t) \in \mathbb{R}^{2} / s(t)<x<r(t), t>0\right\}$.

$x=s(t)$ and $x=r(t)$ are the functions that characterize the free boundaries of the mushy zone. We also follow [10] 
in making the following assumptions on the structure of the mushy zone, which is considered as isothermal:

(1) The material contains a fixed portion of the total latent heat per unit mass (see condition (3)).

(2) Its width is inversely proportional to the gradient of temperature (see condition (4)).

Thermal coefficients involved in the solidification process are assumed to be constant. They are

$l>0$ : latent heat by unit mass,

$k>0$ : thermal conductivity,

$\rho>0$ : mass density,

$c>0$ : specific heat,

$0<\epsilon<1$ : one of the two coefficients which characterize the mushy zone,

$\gamma>0$ : one of the two coefficients which characterize the mushy zone,

$q_{0}>0$ : coefficient that characterizes the heat flux at $x=0$,

$h_{0}>0$ : coefficient that characterizes the heat transfer at $x=0$,

$-D_{\infty}<0$ : bulk temperature at $x=0$.

We suppose that five of the six thermal coefficients $l, k, \rho$, $c, \epsilon$, and $\gamma$ of the solid phase are known and that, by means of a change of phase experiment (solidification of the material at its melting temperature), we are able to measure the quantities $q_{0}, h_{0}$, and $-D_{\infty}$.

Encouraged by the recent works $[12,13]$ and with the aim of the simultaneous determination of temperature $T=$ $T(x, t)$, the two free boundaries $x=r(t)$ and $x=s(t)$, and one unknown thermal coefficient among $l, k, \rho, c, \epsilon$, and $\gamma$, we impose an overspecified boundary condition [2] which consists of the specification of a convective condition at the fixed face $x=0$ (see condition (7)) of the material undergoing the phase-change process. This leads us to the following free boundary problem:

$$
\begin{aligned}
& \rho c T_{t}(x, t)-k T_{x x}(x, t)=0 \quad 0<x<s(t), t>0, \\
& T(s(t), t)=0 \quad t>0, \\
& k T_{x}(s(t), t)=\rho l[\epsilon \dot{s}(t)+(1-\epsilon) \dot{r}(t)] \quad t>0, \\
& T_{x}(s(t), t)(r(t)-s(t))=\gamma \quad t>0, \\
& r(0)=s(0)=0, \\
& k T_{x}(0, t)=\frac{q_{0}}{\sqrt{t}} \quad t>0, \\
& k T_{x}(0, t)=\frac{h_{0}}{\sqrt{t}}\left(T(0, t)+D_{\infty}\right) \quad t>0 .
\end{aligned}
$$

This problem was first studied in [11] with a temperature boundary condition at $x=0$ instead of the convective condition (7) considered in this paper. Moreover, the determination of one unknown thermal coefficient for the one-phase
Lamé-Clapeyron-Stefan problem with an overspecified heat flux condition at the fixed face $x=0$ without a mushy zone was done in [14]. Other papers related to determination of thermal coefficients are [15-40].

The goal of this paper is to obtain the explicit solution to the phase-change process (1)-(7) with one unknown thermal coefficient independent of position and time and the necessary and sufficient conditions on data in order to obtain an explicit formula for the unknown thermal coefficient. In addition, we are interested in analysing the relationship between problem (1)-(7) and the phase-change process given by (1)(6) besides the Dirichlet boundary condition overspecified at $x=0$ given by (31) (see below). In particular, we are interested in solving the problem with Dirichlet boundary condition through problem with convective boundary condition when large values of the coefficient $h_{0}$ that characterizes the heat transfer at $x=0$ are considered.

The organization of the paper is as follows. In Section 2 we prove a preliminary result where necessary and sufficient conditions on data for the phase-change process (1)-(7) are given in order to obtain the temperature $T=T(x, t)$ and the two free boundaries $x=r(t)$ and $x=s(t)$. Based on this preliminary result, in Section 3 , we present and solve six different cases for the phase-change process (1)-(7) according to the choice of the unknown thermal coefficient among $l, k$, $\rho, c, \epsilon$, and $\gamma$. In Section 4 we discuss the relationship between the phase-change process (1)-(6) with the Dirichlet boundary condition (31) and the same process with the convective boundary condition (7). We show that temperature $T_{D}=$ $T_{D}(x, t)$, free boundaries $x=r_{D}(t)$ and $x=s_{D}(t)$, and the explicit formula for the unknown thermal coefficient $l, k$, $\rho, c, \epsilon$, or $\gamma$ for the phase-change process (1)-(6) with the Dirichlet condition (31) can be obtained through the phasechange process with convective condition given by (1)-(7) when $h_{0}$ tends to $+\infty$. Explicit formulae for the unknown thermal coefficient for problems (1)-(7) and (1)-(6) and (31), besides restrictions on data that guarantees their validity, are summarized in Tables 1 and 2, respectively.

\section{Explicit Solution to the Phase-Change Process}

The following lemma represents the base on which the work in this section will be structured.

Lemma 1. The solution to problem (1)-(7) is given by

$$
\begin{aligned}
& T(x, t)=\frac{q_{0} \sqrt{\pi \alpha}}{k}\left[\operatorname{erf}\left(\frac{x}{2 \sqrt{\alpha t}}\right)-\operatorname{erf}(\xi)\right] \\
& \qquad 0<x<s(t), t>0, \\
& s(t)=2 \xi \sqrt{\alpha t} \quad t>0, \\
& r(t)=2\left[\xi+\frac{\gamma k}{2 q_{0} \sqrt{\alpha}} \exp \left(\xi^{2}\right)\right] \sqrt{\alpha t} \quad t>0
\end{aligned}
$$


TABLE 1: Formulae for problem (1)-(7). Explicit formulae for the unknown thermal coefficient $l, \gamma, \epsilon, k, \rho$, or $c$ and coefficient $\xi$ (or the equation that it must satisfy) and the corresponding restrictions on data that guarantee their validity.

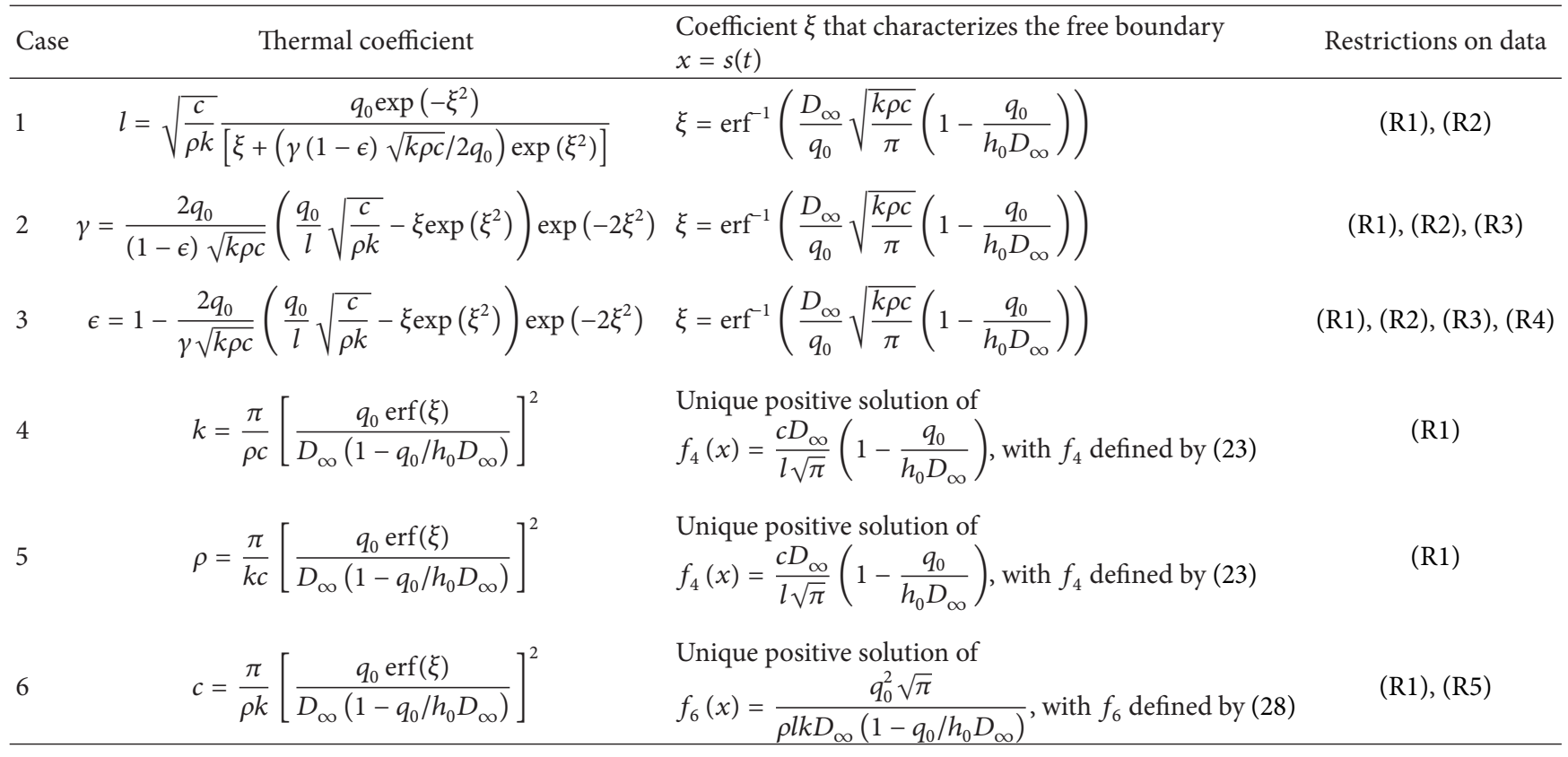

TABLE 2: Formulae for problem (1)-(6) and (31). Explicit formulae for the unknown thermal coefficient $l, \gamma, \epsilon, k, \rho$, or $c$ and coefficient $\xi$ (or the equation that it must satisfy) and the corresponding restrictions on data that guarantee their validity.

\begin{tabular}{|c|c|c|c|}
\hline Case & Thermal coefficient & $\begin{array}{l}\text { Coefficient } \xi \text { that characterizes the free boundary } \\
x=s_{D}(t)\end{array}$ & Restrictions on data \\
\hline 1 & $l=\sqrt{\frac{c}{\rho k}} \frac{q_{0} \exp \left(-\xi^{2}\right)}{\left[\xi+\left(\gamma(1-\epsilon) \sqrt{k \rho c} / 2 q_{0}\right) \exp \left(\xi^{2}\right)\right]}$ & $\xi=\operatorname{erf}^{-1}\left(\frac{D_{\infty}}{q_{0}} \sqrt{\frac{k \rho c}{\pi}}\right)$ & (R6) \\
\hline 2 & $\gamma=\frac{2 q_{0}}{(1-\epsilon) \sqrt{k \rho c}}\left(\frac{q_{0}}{l} \sqrt{\frac{c}{\rho k}}-\xi \exp \left(\xi^{2}\right)\right) \exp \left(-2 \xi^{2}\right)$ & $\xi=\operatorname{erf}^{-1}\left(\frac{D_{\infty}}{q_{0}} \sqrt{\frac{k \rho c}{\pi}}\right)$ & (R7) \\
\hline 3 & $\epsilon=1-\frac{2 q_{0}}{\gamma \sqrt{k \rho c}}\left(\frac{q_{0}}{l} \sqrt{\frac{c}{\rho k}}-\xi \exp \left(\xi^{2}\right)\right) \exp \left(-2 \xi^{2}\right)$ & $\xi=\operatorname{erf}^{-1}\left(\frac{D_{\infty}}{q_{0}} \sqrt{\frac{k \rho c}{\pi}}\right)$ & (R7), (R8) \\
\hline 4 & $k=\frac{\pi}{\rho c}\left[\frac{q_{0} \operatorname{erf}(\xi)}{D_{\infty}}\right]^{2}$ & $\begin{array}{l}\text { Unique positive solution of } \\
F_{4}(x)=\frac{c D_{\infty}}{l \sqrt{\pi}} \text {, with } F_{4} \text { defined by (36) }\end{array}$ & \\
\hline 5 & $\rho=\frac{\pi}{k c}\left[\frac{q_{0} \operatorname{erf}(\xi)}{D_{\infty}}\right]^{2}$ & $\begin{array}{l}\text { Unique positive solution of } \\
F_{4}(x)=\frac{c D_{\infty}}{l \sqrt{\pi}} \text {, with } F_{4} \text { defined by (36) }\end{array}$ & \\
\hline 6 & $c=\frac{\pi}{\rho k}\left[\frac{q_{0} \operatorname{erf}(\xi)}{D_{\infty}}\right]^{2}$ & $\begin{array}{l}\text { Unique positive solution of } \\
F_{6}(x)=\frac{q_{0}^{2} \sqrt{\pi}}{\rho l k D_{\infty}} \text {, with } F_{6} \text { defined by }(37)\end{array}$ & (R9) \\
\hline
\end{tabular}

if and only if the parameters involved in problem (1)-(7) satisfy the following two equations:

$$
\begin{aligned}
& {\left[\xi+\frac{\gamma k(1-\epsilon)}{2 q_{0} \sqrt{\alpha}} \exp \left(\xi^{2}\right)\right] \exp \left(\xi^{2}\right)=\frac{q_{0}}{\rho l \sqrt{\alpha}},} \\
& \operatorname{erf}(\xi)=\frac{k \mathrm{D}_{\infty}}{q_{0} \sqrt{\pi \alpha}}\left(1-\frac{q_{0}}{h_{0} D_{\infty}}\right),
\end{aligned}
$$

where $\alpha=k / \rho$ c represents the thermal diffusivity.
Proof. The kind of phase-change processes considered in this paper has the following general solution [10-12]:

$$
\begin{aligned}
T(x, t) & =A+B \operatorname{erf}\left(\frac{x}{2 \sqrt{\alpha t}}\right) \quad 0<x<s(t), t>0, \\
s(t) & =2 \xi \sqrt{\alpha t} \quad t>0, \\
r(t) & =2 \mu \sqrt{\alpha t} \quad t>0,
\end{aligned}
$$


where coefficients $A, B, \xi$, and $\mu$ depend on the particular specifications of the phase-change process.

In order to have the solution to problem (1)-(7), we impose conditions (2)-(4), (6) and (7) on (11) and obtain that coefficients $A, B$, and $\mu$ must be given by

$$
\begin{aligned}
& A=-\frac{q_{0} \sqrt{\pi \alpha}}{k} \operatorname{erf}(\xi), \\
& B=\frac{q_{0} \sqrt{\pi \alpha}}{k}, \\
& \mu=\xi+\frac{\gamma k \exp \left(\xi^{2}\right)}{2 q_{0} \sqrt{\alpha}},
\end{aligned}
$$

which corresponds to solution (8), and the parameters involved in the problem must satisfy (9) and (10).

As a consequence of Lemma 1, we know that we can solve the phase-change process (1)-(7) with one unknown thermal coefficient through the determination of parameter $\xi$ that characterizes one of the two free boundaries of the mushy zone and the unknown thermal coefficient among $l, k, \rho, c$, $\epsilon$, and $\gamma$. In addition, we also know from Lemma 1 that we can do that by solving the system of (9)-(10).

\section{Explicit Formula for the Unknown Thermal Coefficient}

In this section we present and solve six different cases for the phase-change process (1)-(7) according to the choice of the unknown thermal coefficient among $l, k, \rho, c, \epsilon$, and $\gamma$.

With the aim of organizing our work, we classify each case by making reference to the coefficients which is necessary to know in order to solve it (see Lemma 1):

Case 1: determination of $l$ and $\xi$.

Case 2: determination of $\gamma$ and $\xi$.

Case 3: determination of $\epsilon$ and $\xi$.

Case 4: determination of $k$ and $\xi$.

Case 5: determination of $\rho$ and $\xi$.

Case 6: determination of $c$ and $\xi$.

In addition, with the goal of making our presentation more readable, in the following statements and proofs we introduce several functions. We name these functions with a subscript according the case where they arise.

Theorem 2 (Case 1: determination of $l$ and $\xi$ ). If in problem (1)-(7) one considers the thermal parameter $l$ as an unknown, then its solution is given by (8) with $l$ and $\xi$ given by

$$
\begin{aligned}
l & =\sqrt{\frac{c}{\rho k}} \frac{q_{0} \exp \left(-\xi^{2}\right)}{\left[\xi+\left(\gamma(1-\epsilon) \sqrt{k \rho c} / 2 q_{0}\right) \exp \left(\xi^{2}\right)\right]}, \\
\xi & =\operatorname{erf}^{-1}\left(\frac{D_{\infty}}{q_{0}} \sqrt{\frac{k \rho c}{\pi}}\left(1-\frac{q_{0}}{h_{0} D_{\infty}}\right)\right)
\end{aligned}
$$

if and only if the parameters $q_{0}, h_{0}, D_{\infty}, k, \rho$, and c satisfy the following two inequalities:

$$
\begin{array}{r}
1-\frac{q_{0}}{h_{0} D_{\infty}}>0 \\
\frac{D_{\infty}}{q_{0}} \sqrt{\frac{k \rho c}{\pi}}\left(1-\frac{q_{0}}{h_{0} D_{\infty}}\right)<1 .
\end{array}
$$

Proof. Due to properties of the error function, it follows that a necessary and sufficient condition for the existence and uniqueness of a positive solution to (10) is

$$
0<\frac{D_{\infty}}{q_{0}} \sqrt{\frac{k \rho c}{\pi}}\left(1-\frac{q_{0}}{h_{0} D_{\infty}}\right)<1,
$$

which is equivalent to inequalities (R1) and (R2). In that case, the positive solution to (10) is given by (14). Finally, it follows from (9) that $l$ is the positive thermal coefficient given by (13).

Theorem 3 (Case 2: determination of $\gamma$ and $\xi$ ). If in problem (1)-(7) one considers the thermal parameter $\gamma$ as an unknown, then its solution is given by (8) with $\gamma$ given by

$$
\gamma=\frac{2 q_{0}}{(1-\epsilon) \sqrt{k \rho c}}\left(\frac{q_{0}}{l} \sqrt{\frac{c}{\rho k}}-f_{2}(\xi)\right) \exp \left(-2 \xi^{2}\right)
$$

and $\xi$ given by (14), if and only if the parameters $q_{0}, h_{0}, D_{\infty}, k$, $\rho, c$, and $l$ satisfy inequalities (R1) and (R2) and

$$
f_{2}\left(\operatorname{erf}^{-1}\left(\frac{D_{\infty}}{q_{0}} \sqrt{\frac{k \rho c}{\pi}}\left(1-\frac{q_{0}}{h_{0} D_{\infty}}\right)\right)\right)<\frac{q_{0}}{l} \sqrt{\frac{c}{\rho k}},
$$

where the real function $f_{2}$ is defined by

$$
f_{2}(x)=x \exp \left(x^{2}\right), \quad x>0 .
$$

Proof. As we see in the proof of Theorem 2, a necessary and sufficient condition that guarantees the existence and uniqueness of solution to (10) is that inequalities (R1) and (R2) hold, and, in that case, the coefficient $\xi$ is given by (14).

On the other hand, it follows from (9) that $\gamma$ is given by (16). This coefficient is positive if and only if

$$
f_{2}(\xi)<\frac{q_{0}}{l} \sqrt{\frac{c}{\rho k}},
$$

where $f_{2}$ is the real function defined in (17). Taking into account the expression of $\xi$ given in (14), we have that inequality (18) is equivalent to inequality (R3).

Theorem 4 (Case 3: determination of $\epsilon$ and $\xi$ ). If in problem (1)-(7) one considers the thermal parameter $\epsilon$ as an unknown, then its solution is given by (8) with $\epsilon$ given by

$$
\epsilon=1-\frac{2 q_{0}}{\gamma \sqrt{k \rho c}}\left(\frac{q_{0}}{l} \sqrt{\frac{c}{\rho k}}-f_{2}(\xi)\right) \exp \left(-2 \xi^{2}\right)
$$


and $\xi$ given by (14), if and only if the parameters $q_{0}, h_{0}, D_{\infty}, k$, $\rho, c$, and $\gamma$ satisfy inequalities (R1), (R2), and (R3) and

$$
\begin{aligned}
& \frac{\gamma \sqrt{k \rho c}}{2 q_{0}} \\
& \cdot \exp \left(2\left[\operatorname{erf}^{-1}\left(\frac{D_{\infty}}{q_{0}} \sqrt{\frac{k \rho c}{\pi}}\left(1-\frac{q_{0}}{h_{0} D_{\infty}}\right)\right)\right]^{2}\right) \\
& +f_{2}\left(\operatorname{erf}^{-1}\left(\frac{D_{\infty}}{q_{0}} \sqrt{\frac{k \rho c}{\pi}}\left(1-\frac{q_{0}}{h_{0} D_{\infty}}\right)\right)\right)>\frac{q_{0}}{l} \\
& \cdot \sqrt{\frac{c}{\rho k}},
\end{aligned}
$$

where $f_{2}$ is the real function defined in (17).

Proof. Conditions (R1) and (R2) and the expression of $\xi$ given in (14) arise in the same way as that in the precedent proofs. On the other hand, it follows from (9) that $\epsilon$ is given by (19), $f_{2}$ being the real function defined in (17). This coefficient is positive if and only if

$$
\frac{2 q_{0}}{\gamma \sqrt{k \rho c}}\left(\frac{q_{0}}{l} \sqrt{\frac{c}{\rho k}}-f_{2}(\xi)\right) \exp \left(-2 \xi^{2}\right)<1 .
$$

Taking into account the expression of $\xi$ given in (14), we have that inequality (20) is equivalent to inequality (R4). Finally, we have that $\epsilon$ given in (19) is less than 1 if and only if $f_{2}(\xi)<q_{0} / \rho l \sqrt{\alpha}$, which, as we see in the proof of Theorem 3 , is equivalent to condition (R3).

Theorem 5 (Case 4: determination of $k$ and $\xi$ ). If in problem (1)-(7) one considers the thermal parameter $k$ as an unknown, then its solution is given by (8) with $k$ given by

$$
k=\frac{\pi}{\rho c}\left[\frac{q_{0} \operatorname{erf}(\xi)}{D_{\infty}\left(1-q_{0} / h_{0} D_{\infty}\right)}\right]^{2}
$$

and $\xi$ is the unique solution to the equation

$$
f_{4}(x)=\frac{c D_{\infty}}{l \sqrt{\pi}}\left(1-\frac{q_{0}}{h_{0} D_{\infty}}\right), \quad x>0,
$$

where $f_{4}$ is the real function defined by

$$
\begin{aligned}
& f_{4}(x)=\left[x+\frac{\gamma \sqrt{\pi}(1-\epsilon)}{2 D_{\infty}\left(1-q_{0} / h_{0} D_{\infty}\right)} \operatorname{erf}(x) \exp \left(x^{2}\right)\right] \\
& \cdot \operatorname{erf}(x) \exp \left(x^{2}\right), \quad x>0
\end{aligned}
$$

if and only if the parameters $q_{0}, h_{0}$, and $D_{\infty}$ satisfy inequality (R1).

Proof. The system of (9)-(10) is equivalent to

$$
\begin{aligned}
\sqrt{k} & =\sqrt{\frac{\pi}{\rho c}} \frac{q_{0} \operatorname{erf}(\xi)}{D_{\infty}\left(1-q_{0} / h_{0} D_{\infty}\right)}, \\
f_{4}(\xi) & =\frac{c D_{\infty}}{l \sqrt{\pi}}\left(1-\frac{q_{0}}{h_{0} D_{\infty}}\right),
\end{aligned}
$$

where $f_{4}$ is the real function defined in (23). A necessary condition for existence of solution to this system is that inequality (R1) holds. Then, if we assume that (R1) holds, we immediately obtain that $k$ is given by (21). To complete the proof it only remains to demonstrate that (22) admits a unique positive solution. This follows from the fact that $f_{4}$ is an increasing function such that $f\left(0^{+}\right)=0$ and $f(+\infty)=+\infty$.

Theorem 6 (Case 5: determination of $\rho$ and $\xi$ ). If in problem (1)-(7) one considers the thermal parameter $\rho$ as an unknown, then its solution is given by (8) with $\rho$ given by

$$
\rho=\frac{\pi}{k c}\left[\frac{q_{0} \operatorname{erf}(\xi)}{D_{\infty}\left(1-q_{0} / h_{0} D_{\infty}\right)}\right]^{2}
$$

and $\xi$ is the unique solution to (22), if and only if the parameters $q_{0}, h_{0}$, and $D_{\infty}$ satisfy inequality (R1).

Proof. It is similar to the proof of Theorem 5.

Theorem 7 (Case 6: determination of $c$ and $\xi$ ). If in problem (1)-(7) one considers the thermal parameter $c$ as an unknown, then its solution is given by (8) with $c$ given by

$$
c=\frac{\pi}{\rho k}\left[\frac{q_{0} \operatorname{erf}(\xi)}{D_{\infty}\left(1-q_{0} / h_{0} D_{\infty}\right)}\right]^{2}
$$

and $\xi$ is the unique solution to the equation

$$
f_{6}(x)=\frac{q_{0}^{2} \sqrt{\pi}}{\rho l k D_{\infty}\left(1-q_{0} / h_{0} D_{\infty}\right)}, \quad x>0,
$$

where $f_{6}$ is the real function defined by

$$
\begin{aligned}
f_{6}(x)= & {\left[\frac{x}{\operatorname{erf}(x)}+\frac{\gamma \sqrt{\pi}(1-\epsilon)}{2 D_{\infty}\left(1-q_{0} / h_{0} D_{\infty}\right)} \exp \left(x^{2}\right)\right] } \\
& \cdot \exp \left(x^{2}\right), \quad x>0,
\end{aligned}
$$

if and only if the parameters $q_{0}, h_{0}$, and $D_{\infty}$ satisfy inequality (R1) and

$$
1-\frac{q_{0}}{h_{0} D_{\infty}}<\frac{1}{D_{\infty}}\left[\frac{2 q_{0}^{2}}{\rho l k}-\gamma(1-\epsilon)\right] .
$$

Proof. The system of (9)-(10) is equivalent to

$$
\begin{aligned}
\sqrt{c} & =\sqrt{\frac{\pi}{\rho k}} \frac{q_{0} \operatorname{erf}(\xi)}{D_{\infty}\left(1-q_{0} / h_{0} D_{\infty}\right)}, \\
f_{6}(\xi) & =\frac{q_{0}^{2} \sqrt{\pi}}{\rho l k D_{\infty}\left(1-q_{0} / h_{0} D_{\infty}\right)},
\end{aligned}
$$

where $f_{6}$ is the real function defined in (28). A necessary condition for existence of solution to this system is that inequality (R1) holds. Then, if we assume that (R1) holds, we immediately obtain that $c$ is given by (26). To complete the proof it only remains to demonstrate that (27) admits a unique positive solution. Since $f_{6}$ is an increasing function such that 
$f\left(0^{+}\right)=\pi / 2+\gamma(1-\epsilon) \sqrt{\pi} / 2 D_{\infty}\left(1-q_{0} / h_{0} D_{\infty}\right)$ and $f(+\infty)=$ $+\infty$, it follows that a necessary and sufficient condition for existence (and uniqueness) of solution to (27) is that

$$
\frac{\pi}{2}+\frac{\gamma(1-\epsilon) \sqrt{\pi}}{2 D_{\infty}\left(1-q_{0} / h_{0} D_{\infty}\right)}<\frac{q_{0}^{2} \sqrt{\pi}}{\rho l k D_{\infty}\left(1-q_{0} / h_{0} D_{\infty}\right)}
$$

which is equivalent to inequality (R5).

Table 1 summarizes the results of this section, corresponding to 6 cases.

\section{The Phase-Change Process with Large Heat Transfer Coefficient}

A similar phase-change process to (1)-(7) with one unknown thermal coefficient has been studied in [11]. In that paper, the author considers a fusion process with a mushy zone given by (1)-(6) overspecified with a temperature boundary condition and obtains the explicit solution to some cases. Encouraged by [11], we consider the solidification process (1)-(6) with one unknown thermal coefficient overspecified with the Dirichlet boundary condition:

$$
T(0, t)=-D_{\infty}, \quad t>0
$$

We can see this condition as the limit case of the convective boundary condition (7) when the heat transfer coefficient $h_{0}$ tends to $+\infty$. From a physical point of view, overspecifying the phase-change process (1)-(6) by imposing the convective boundary condition (7) seems to be more appropriate than imposing the Dirichlet condition (31). This section is devoted to showing that the temperature $T_{D}=T_{D}(x, t)$, the free boundaries $x=r_{D}(t)$ and $x=s_{D}(t)$, and the explicit formula for the unknown thermal coefficient $l, k, \rho, c, \epsilon$, or $\gamma$ for the phase-change process with Dirichlet boundary condition given by (1)-(6) and (31) can be obtained through the phasechange process with convective boundary condition given by (1)-(7) when $h_{0}$ tends to $+\infty$.

We begin with a result related to the solution to the phasechange process (1)-(6) and (31). This result may be shown in much the same manner as Lemma 1; thus we do not give its proof here.

Lemma 8. The solution to problem (1)-(6) and (31) is given by (8); that is,

$$
\begin{aligned}
T_{D}(x, t) & =\frac{q_{0} \sqrt{\pi \alpha}}{k}\left[\operatorname{erf}\left(\frac{x}{2 \sqrt{\alpha t}}\right)-\operatorname{erf}(\xi)\right] \\
& 0<x<s_{D}(t), t>0, \\
s_{D}(t) & =2 \xi \sqrt{\alpha t} \quad t>0, \\
r_{D}(t) & =2\left[\xi+\frac{\gamma k}{2 q_{0} \sqrt{\alpha}} \exp \left(\xi^{2}\right)\right] \sqrt{\alpha t} \quad t>0,
\end{aligned}
$$

where $\alpha=k / \rho$ c represents the thermal diffusivity, if and only if the parameters involved in problem (1)-(6) and (31) satisfy the following two equations:

$$
\begin{aligned}
& {\left[\xi+\frac{\gamma(1-\epsilon) \sqrt{\pi}}{2 D_{\infty}} \operatorname{erf}(\xi) \exp \left(\xi^{2}\right)\right] \operatorname{erf}(\xi) \exp \left(\xi^{2}\right)} \\
& =\frac{c D_{\infty}}{l \sqrt{\pi}}, \\
& \operatorname{erf}(\xi)=\frac{D_{\infty}}{q_{0}} \sqrt{\frac{k \rho c}{\pi}} .
\end{aligned}
$$

From Lemma 8 we know that, in order to have temperature $T_{D}=T_{D}(x, t)$, the free boundaries $x=r_{D}(t)$ and $x=$ $s_{D}(t)$, and the unknown thermal coefficient $l, k, \rho, c, \epsilon$, or $\gamma$ for problem (1)-(6) and (31), it is enough to find the unknown thermal coefficient and the parameter that characterizes the free boundary $s_{D}(t)$. Proceeding analogously to the work done in [11] or in Section 3, we can obtain the thermal coefficient $l, k, \rho, c, \epsilon$, or $\gamma$ and the parameter $\xi$ for problem (1)-(6) and (31). Formulae for those quantities, besides restrictions on data that guarantee their validity, are summarized in Table 2 (restrictions on data and definitions on functions mentioned in Table 2 are listed as follows).

List of restrictions on data for problem (1)-(6) and (31) mentioned in Table 2 is

$$
\begin{aligned}
& \frac{D_{\infty}}{q_{0}} \sqrt{\frac{k \rho c}{\pi}}<1, \\
& \frac{D_{\infty}}{q_{0}} \sqrt{\frac{k \rho c}{\pi}}<\operatorname{erf}(\eta),
\end{aligned}
$$

where $\eta$ is the unique positive solution to the equation

$$
f_{2}(x)=\frac{q_{0}}{l} \sqrt{\frac{c}{\rho k}}, \quad x>0,
$$

$f_{2}$ being the real function defined in (17);

$$
\frac{D_{\infty}}{q_{0}} \sqrt{\frac{k \rho c}{\pi}}>\operatorname{erf}(\eta), \quad x>0,
$$

where $\eta$ is the unique positive solution to the equation

$$
f_{2}(x)+\frac{\gamma \sqrt{k \rho c}}{2 q_{0}} \exp \left(2 x^{2}\right)=\frac{q_{0}}{l} \sqrt{\frac{c}{k \rho}}, \quad x>0,
$$

$f_{2}$ being the real function defined in (17);

$$
\frac{l k \rho D_{\infty}}{2 q_{0}}\left(1+\frac{\gamma(1-\epsilon)}{D_{0}}\right)<1
$$

List of definitions of functions related to problem (1)-(6) and (31) mentioned in Table 2 is

$$
\begin{aligned}
F_{4}(x)= & \operatorname{erf}(x) f_{2}(x) \\
& +\frac{(1-\epsilon) \gamma \sqrt{\pi}}{2 D_{\infty}}[\operatorname{erf}(x)]^{2} \exp \left(2 x^{2}\right), \quad x>0,
\end{aligned}
$$


$f_{2}$ being the real function defined in (17);

$$
\begin{array}{r}
F_{6}(x)=\left(\frac{x}{\operatorname{erf}(x)}+\frac{\gamma(1-\epsilon) \sqrt{\pi}}{2 D_{\infty}} \exp \left(x^{2}\right)\right) \exp \left(x^{2}\right), \\
x>0 .
\end{array}
$$

On the other hand, it is not difficult to verify that formulae and restrictions on data given in Table 2 correspond to formulae and restrictions on data given in Table 1 for $h_{0}$ tending to $+\infty$. This fact, besides Lemmas 1 and 8 , allows us to conclude that we can solve the phase-change process (1)-(6) with one unknown thermal coefficient overspecified by the Dirichlet condition (31) through the phase-change process (1)-(7), which is overspecified by the more physically appropriate convective boundary condition (7), when the heat transfer coefficient $h_{0}$ tends to $+\infty$.

\section{Conclusions}

In this paper, we consider a semi-infinite material under a solidification process with a mushy zone caused by an initial heat flux boundary condition, when the thermophysical parameters involved in the phase-change process are assumed to be constant. We solve the associated free boundary problem overspecified with a convective boundary condition and obtain the temperature, the two free boundaries of the mushy zone and one thermal coefficient among the latent heat by unit mass, the thermal conductivity, the mass density, the specific heat, and the two coefficients that characterize the mushy zone, when the bulk temperature and the coefficients that characterize the heat flux and the heat transfer at the boundary are assumed to be known. As a consequence, we give formulae for the temperature, the two free boundaries, and the unknown thermal coefficient, besides necessary and sufficient conditions on data, in order to obtain them. In addition, we present the relationship between the phase-change process studied in this paper and another similar phasechange process which is overspecified by a Dirichelt boundary condition. From this relationship, we solve the problem with the Dirichlet condition by considering a large heat transfer coefficient in the problem with the convective condition. In this way, we solve the phase-change process overspecified with a temperature boundary condition through the more physically appropriate phase-change problem overspecified with a convective boundary condition. We summarize explicit formulae for the unknown thermal coefficient for both problems in Tables 1 and 2.

\section{Conflict of Interests}

The authors declare that there is no conflict of interests regarding the publication of this paper.

\section{Acknowledgments}

This paper has been partially sponsored by the Project PIP no. 0534 from CONICET-UA (Rosario, Argentina) and AFOSRSOARD Grant FA 9550-14-1-0122. The authors like to thank two anonymous referees for the helpful comments.

\section{References}

[1] V. Alexiades and A. D. Solomon, Mathematical Modeling of Melting and Freezing Processes, Hemisphere Publishing Corporation, Washington, DC, USA, 1993.

[2] J. R. Cannon, The One-Dimensional Heat Equation, AddisonWesley, Menlo Park, Calif, USA, 1984.

[3] H. S. Carslaw and J. C. Jaeger, Conduction of Heat in Solids, The Clarendon Press, Oxford, UK, 1959.

[4] J. Crank, Free and Moving Boundary Problems, The Clarendon Press, Oxford, UK, 1984.

[5] A. Fasano, Mathematical Models of Some Diffusive Processes with Free Boundaries, vol. 11 of MAT Serie A: Conferencias, Seminarios Y Trabajos de Matematica, 2005.

[6] S. C. Gupta, The Classical Stefan Problem. Basic Concepts, Modelling and Analysis, Elsevier, Amsterdam, The Netherlands, 2003.

[7] J. M. Hill, One-Dimensional Stefan Problems: An Introduction, Longman Higher Education, Essex, UK, 1987.

[8] V. J. Lunardini, Heat Transfer with Freezing and Thawing, Elsevier Science Publisher BV, 1991.

[9] A. B. Tayler, Mathematical Models in Applied Mechanics, Clarendon Press, Oxford, UK, 1986.

[10] A. D. Solomon, D. G. Wilson, and V. Alexiades, "A mushy zone model with an exact solution," Letters in Heat and Mass Transfer, vol. 9, no. 4, pp. 319-324, 1982.

[11] D. A. Tarzia, "Determination of unknown thermal coefficients of a semi-infinite material for the one-phase Lamé-Clapeyron (Stefan) problem through the Solomon-Wilson-Alexiades' mushy zone model," International Communications in Heat and Mass Transfer, vol. 14, no. 2, pp. 219-228, 1987.

[12] D. A. Tarzia, "Explicit solutions for the Solomon-WilsonAlexiades's mushy zone model with convective or heat flux boundary conditions," http://arxiv.org/abs/1503.02736.

[13] D. Tarzia, "Relationship between Neumann solutions for twophase Lamé-Clapeyron-Stefan problems with convective and temperature boundary conditions," Thermal Science, 12 pages, 2015.

[14] D. A. Tarzia, "Determination of the unknown coefficients in the Lamé-Clapeyron Stefan problem (or one-phase Stefan problem)," Advances in Applied Mathematics, vol. 3, no. 1, pp. 74-82, 1982.

[15] V. L. Baranov, A. A. Zasyad'ko, and G. A. Frolov, "Integrodifferential method of solving the inverse coefficient heat conduction problem," Journal of Engineering Physics and Thermophysics, vol. 83, no. 1, pp. 60-71, 2010.

[16] I. Boulaoued and A. Mhimid, "Determination of the diffusion coefficient of new insulators composed of vegetable fibers," Thermal Science, vol. 16, no. 4, pp. 987-995, 2012.

[17] R. Das, S. C. Mishra, and R. Uppaluri, "Retrieval of thermal properties in a transient conduction-radiation problem with variable thermal conductivity," International Journal of Heat and Mass Transfer, vol. 52, no. 11-12, pp. 2749-2758, 2009.

[18] Z.-C. Deng, L. Yang, J.-N. Yu, and G.-W. Luo, "An inverse problem of identifying the coefficient in a nonlinear parabolic equation," Nonlinear Analysis: Theory, Methods \& Applications, vol. 71, no. 12, pp. 6212-6221, 2009.

[19] A. Erdem, D. Lesnic, and A. Hasanov, "Identification of a spacewise dependent heat source," Applied Mathematical Modelling, vol. 37, no. 24, pp. 10231-10244, 2013. 
[20] N. L. Gol'dman, "One-phase inverse stefan problems with unknown nonlinear sources," Differential Equations, vol. 49, no. 6, pp. 680-687, 2013.

[21] A. Hazanee, M. I. Ismailov, D. Lesnic, and N. B. Kerimov, "An inverse time-dependent source problem for the heat equation," Applied Numerical Mathematics, vol. 69, pp. 13-33, 2013.

[22] A. Hasanov and B. Pektas, "Identification of an unknown time-dependent heat source term from overspecified Dirichlet boundary data by conjugate gradient method," Computers \& Mathematics with Applications, vol. 65, no. 1, pp. 42-57, 2013.

[23] E. Hetmaniok, I. Nowak, D. Slota, R. Witula, and A. Zielonka, "Solution of the inverse heat conduction problem with Neumann boundary condition by using the homotopy perturbation method," Thermal Science, vol. 17, no. 3, pp. 643-650, 2013.

[24] N. Hryntsiv, "The inverse problem with free boundary for a weakly degenerate parabolic equation," Journal of Mathematical Sciences, vol. 183, no. 6, pp. 779-795, 2012.

[25] M. Hussein and D. Lesnic, "Determination of a time-dependent thermal diffusivity and free boundary in heat conduction," International Communications in Heat and Mass Transfer, vol. 53, pp. 154-163, 2014.

[26] M. S. Hussein, D. Lesnic, and M. I. Ivanchov, "Simultaneous determination of time-dependent coefficients in the heat equation," Computers \& Mathematics with Applications, vol. 67, no. 5, pp. 1065-1091, 2014.

[27] Y. Inatomi, F. Onishi, K. Nagashio, and K. Kuribayashi, "Density and thermal conductivity measurements for silicon melt by electromagnetic levitation under a static magnetic field," International Journal of Thermophysics, vol. 28, no. 1, pp. 44-59, 2007.

[28] F. Kanca and M. I. Ismailov, "The inverse problem of finding the time-dependent diffusion coefficient of the heat equation from integral overdetermination data," Inverse Problems in Science and Engineering, vol. 20, no. 4, pp. 463-476, 2012.

[29] N. B. Kerimov and M. I. Ismailov, "An inverse coefficient problem for the heat equation in the case of nonlocal boundary conditions," Journal of Mathematical Analysis and Applications, vol. 396, no. 2, pp. 546-554, 2012.

[30] M. Lamvik and J. M. Zhou, "A novel technique for measuring the thermal conductivity of metallic materials during melting and solidification," Measurement Science and Technology, vol. 6, no. 7, pp. 880-887, 1995.

[31] G. Li, D. Yao, Y. Wang, and X. Jia, "A nonlinear solute transport model and data reconstruction with parameter determination in an undisturbed soil-column experiment," Mathematical Problems in Engineering, vol. 2011, Article ID 679531, 14 pages, 2011.

[32] T. Lu, W. Han, P. Jiang, Y. Zhu, J. Wu, and C. Liu, "A twodimensional inverse heat conduction problem for simultaneous estimation of heat convection coefficient, fluid temperature and wall temperature on the inner wall of a pipeline," Progress in Nuclear Energy, vol. 81, pp. 161-168, 2015.

[33] W. Peng, J.-M. Zhou, Y. Li, Y. Yang, and M.-R. Guo, "A dynamic technique for the measurement of thermal conductivity of molten salt based on cylindrical melting model," Journal of Thermal Analysis and Calorimetry, vol. 115, no. 2, pp. 1767-1777, 2014.

[34] N. N. Salva and D. A. Tarzia, "A sensitivity analysis for the determination of unknown thermal coefficients through a phase-change process with temperature-dependent thermal conductivity," International Communications in Heat and Mass Transfer, vol. 38, no. 4, pp. 418-424, 2011.
[35] E. A. Santillan Marcus and D. A. Tarzia, "Determination of one unknown thermal coefficient of a semi-infinite porous material through a desublimation problem with coupled heat and moisture flows," JP Journal of Heat and Mass Transfer, vol. 1, no. 3, pp. 251-270, 2007.

[36] R. S. Vajjha and D. K. Das, "Experimental determination of thermal conductivity of three nanofluids and development of new correlations," International Journal of Heat and Mass Transfer, vol. 52, no. 21-22, pp. 4675-4682, 2009.

[37] A.-H. Wang, X.-G. Liang, and J.-X. Ren, "Constructal enhancement of heat conduction with phase change," International Journal of Thermophysics, vol. 27, no. 1, pp. 126-138, 2006.

[38] Y. Yang, J.-N. Yu, and Z.-C. Deng, "An inverse problem of identifying the coefficient of parabolic equation," Applied Mathematical Modelling, vol. 32, no. 10, pp. 1984-1995, 2008.

[39] Y. Yang and J. Zhou, "An experimental technique for liquid/solid thermal conductivity measurements at the melting point," International Journal of Thermophysics, vol. 27, no. 1, pp. 184194, 2006.

[40] G. Zhou, B. Wu, W. Ji, and S. Rho, "Time- or space-dependent coefficient recovery in parabolic partial differential equation for sensor array in the biological computing," Mathematical Problems in Engineering, vol. 2015, Article ID 573932, 9 pages, 2015. 


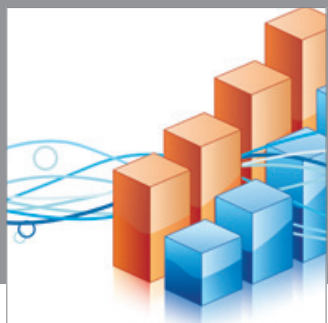

Advances in

Operations Research

mansans

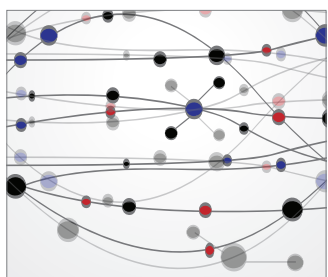

The Scientific World Journal
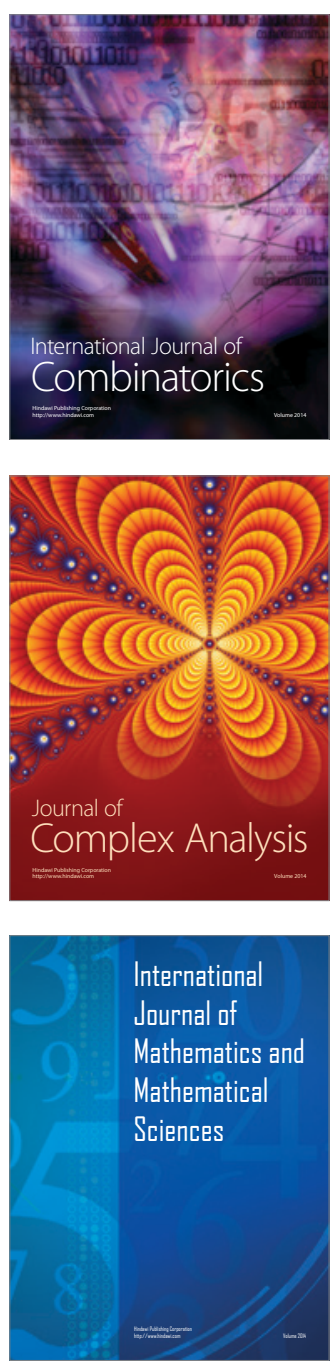
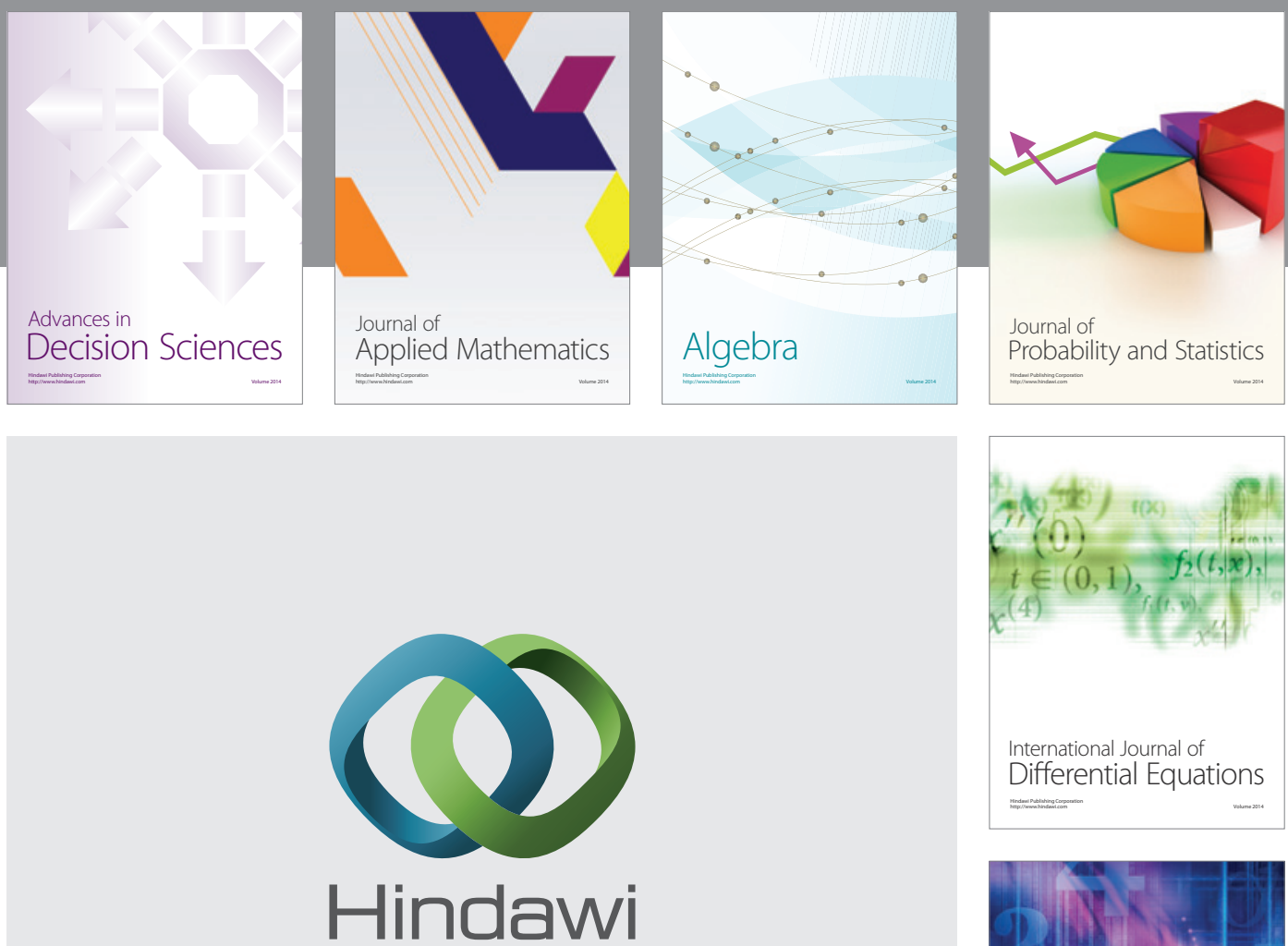

Submit your manuscripts at http://www.hindawi.com
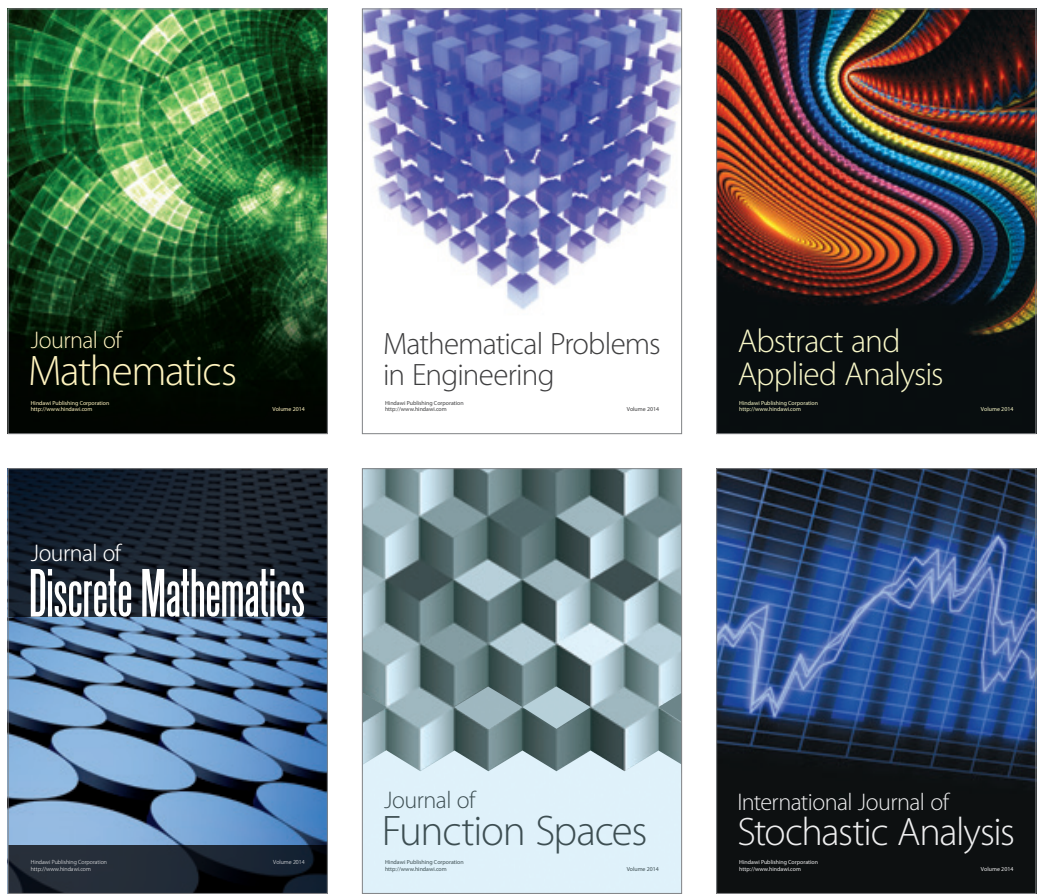

Journal of

Function Spaces

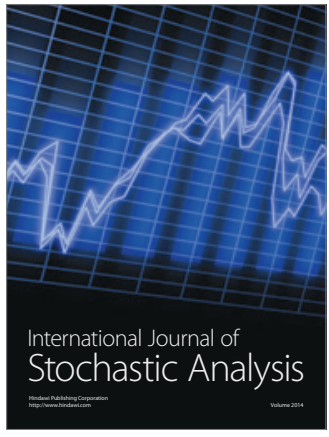

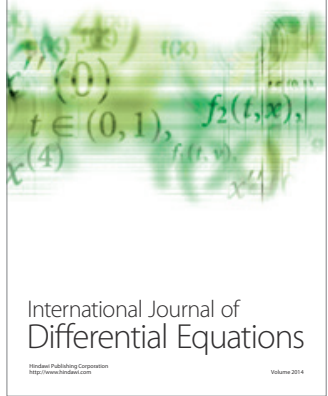
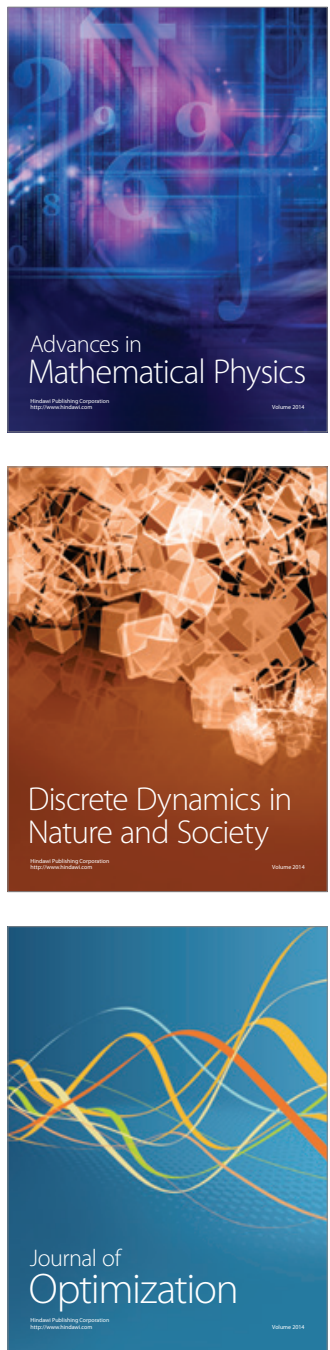